\title{
Continuous variable entanglement using cold atoms
}

\author{
V. Josse, A. Dantan, A. Bramati, M. Pinard, and E. Giacobino \\ Laboratoire Kastler Brossel, Université Pierre et Marie Curie, \\ Case 74, 4 place Jussieu, 75252 Paris Cedex 05, France
}

(Dated: May 31, 2021)

\begin{abstract}
We present an experimental demonstration of both quadrature and polarization entanglement generated via the interaction between a coherent linearly polarized field and cold atoms in a high finesse optical cavity. The non linear atom-field interaction produces two squeezed modes with orthogonal polarizations which are used to generate a pair of non separable beams, the entanglement of which is demonstrated by checking the inseparability criterion for continuous variables recently derived by Duan et al. [Phys. Rev. Lett. 84, 2722 (2000)] and calculating the entanglement of formation [Giedke et al., Phys. Rev. Lett. 91, 107901 (2003)].

PACS numbers: 42.50.Dv, 42.50.Ct, 03.67.Hk
\end{abstract}

Entanglement in the continuous variable regime has attracted a lot of attention in the quantum optics and quantum information fields in connection with quantum teleportation, cryptography, quantum computing and dense coding [1]. Since the first realization of quadrature entangled beams by $\mathrm{Ou}$ et al. 2], various methods, such as $\chi^{(2)}$ process in optical parametric amplifier (OPA) [3], or Kerr effect in optical fibers [4, have been used to generate non separable beams. Recently, the concept of polarization entanglement, i.e. entanglement of Stokes operators between two beams, has been investigated by Korolkova et al. [5], and first demonstrated experimentally by Bowen et al. [6] by mixing two squeezed beams issued from independent OPAs. The Kerr non-linearity of fibers was also exploited by Glöckl et al. to generate a pulsed source of polarization entanglement [7].

In this paper we show evidence for continuous variable entanglement generated using the interaction between a linearly polarized coherent field and a cloud of cold cesium atoms placed in a high finesse optical cavity. We demonstrate the entanglement using the inseparability criterion proposed by Duan et al. and Simon [8]. We generate two kinds of entanglement with the same system, quadrature entanglement and polarization entanglement. For this, we use the recently reported generation of polarization squeezing [9] in the field that has interacted with cold atoms; both the mean field mode and the vacuum mode with orthogonal polarization exiting the cavity can be squeezed. First, we show how a direct measurement of the quadrature entanglement of the beam exiting the cavity can be achieved using two balanced homodyne detections. We then give the form of the covariance matrix and the associated entanglement of formation (EOF), which, for Gaussian symmetric states, is directly related to the inseparability criterion value 10. Last, we produce two non separable beams by mixing two parts of the previous outgoing beam with a strong field and achieve polarization entanglement by locking the relative phases between the strong field and the weak field exiting the cavity.

First, let us consider two orthogonally polarized modes $A_{a}$ and $A_{b}$ of the electromagnetic field satisfying the usual bosonic commutation rules $\left[A_{\alpha}, A_{\beta}^{\dagger}\right]=\delta_{\alpha \beta}$. If $X_{\alpha}(\theta)=\left(A_{\alpha}^{\dagger} e^{i \theta}+A_{\alpha} e^{-i \theta}\right)$ and $Y_{\alpha}(\theta)=X_{\alpha}(\theta+\pi / 2)$ are the usual quadrature operators (rotated in the Fresnel diagram by angle $\theta), X_{a}+X_{b}$ and $Y_{a}-Y_{b}$ are the continuous variable analogous of the EPR-type operators first introduced by Einstein, Podolsky and Rosen [11]. The criterion of [8] sets a limit for inseparability on the sum of the operator variances

$$
\mathcal{I}_{a, b}(\theta)=\frac{1}{2}\left[\Delta^{2}\left(X_{a}+X_{b}\right)(\theta)+\Delta^{2}\left(Y_{a}-Y_{b}\right)(\theta)\right]<2
$$

For states with Gaussian statistics, $\mathcal{I}_{a, b}<2$ is a sufficient condition for entanglement and has already been used in several experiments to demonstrate continuous variable entanglement [5, 6, 7]. Moreover, Giedke et al. recently calculated the EOF of Gaussian symmetric states 10. and showed it to be directly related to the amount of EPR-type correlations given by (1).

In our system, an $x$-polarized beam interacts with a cloud of cold cesium atoms in an optical cavity. The experimental set-up [9] is shown in Fig. [1 We probe the atoms with a linearly polarized laser beam detuned by about $50 \mathrm{MHz}$ in the red of the $6 \mathrm{~S}_{1 / 2}, \mathrm{~F}=4$ to $6 \mathrm{P}_{3 / 2}$, $\mathrm{F}=5$ transition. The optical power of the probe beam ranges from 5 to $15 \mu \mathrm{W}$. After exiting the cavity, both the mean field mode $A_{x}$ and the orthogonally polarized vacuum mode $A_{y}$ are squeezed for frequencies ranging between 3 and $12 \mathrm{MHz}$. An interpretation of these results can be provided by modelling the complicated $6 \mathrm{~S}_{1 / 2}, \mathrm{~F}=4$ to $6 \mathrm{P}_{3 / 2}, \mathrm{~F}=5$ transition by an $\mathrm{X}$-like four-level atomic structure [12]. When the optical transitions are saturated, the atoms behave as a Kerr-like medium for the mean field mode $A_{x}$, which can be squeezed. Furthermore, the orthogonally polarized vacuum mode $A_{y}$ is also squeezed on account of cross-Kerr effect, but for an orthogonal quadrature [9, 12]. 
Our goal is to retrieve the two modes with orthogonal polarizations which exhibit maximal EPR-type correlations according to the inseparability criterion (1). We therefore minimize the quantity $\mathcal{I}_{a, b}(\theta)$ with respect to $a, b$ and $\theta$. Expanding (11) yields

$$
\begin{aligned}
\mathcal{I}_{a, b}(\theta)= & \left\langle\delta A_{a}^{\dagger} \delta A_{a}+\delta A_{a} \delta A_{a}^{\dagger}+\delta A_{b} \delta A_{b}^{\dagger}+\delta A_{b}^{\dagger} \delta A_{b}\right\rangle \\
& +2\left[e^{-2 i \theta}\left\langle\delta A_{a} \delta A_{b}\right\rangle+e^{2 i \theta}\left\langle\delta A_{a}^{\dagger} \delta A_{b}^{\dagger}\right\rangle\right]
\end{aligned}
$$

The right hand side of the first line in (2) is independent of the polarization basis, while the second line can be written as $4 \cos (2 \theta-2 \phi)\left|\left\langle\delta A_{a} \delta A_{b}\right\rangle\right|$, where $2 \phi$ is the phase angle of $\left\langle\delta A_{a} \delta A_{b}\right\rangle$. Minimizing $\mathcal{I}_{a, b}(\theta)$ corresponds to maximizing $\left|\left\langle\delta A_{a} \delta A_{b}\right\rangle\right|$ with respect to $a, b$. In order to find the optimal field components $a, b$, we introduce another polarization basis $u, v$, such that $\left\langle\delta A_{u} \delta A_{v}\right\rangle=0$. It can be shown that there always exists such a polarization basis and that the $u$ and $v$ modes quadrature variances are minimal for the same value of $\theta$ [13]. The optimal correlations produced in the system are directly related to the quantum noise properties of these modes $u, v$. Indeed, the maximally correlated modes $a^{*}, b^{*}$ are

$$
A_{a^{*}}=\left(A_{u}+i A_{v}\right) / \sqrt{2}, \quad A_{b^{*}}=\left(A_{u}-i A_{v}\right) / \sqrt{2}
$$

and the minimum value of $\mathcal{I}_{a, b}$ is then given by the sum of the $u, v$ modes minimal noises

$$
\mathcal{I}_{a^{*}, b^{*}}=\min _{a, b, \theta} \mathcal{I}_{a, b}(\theta)=\min _{\theta}\left[\Delta^{2} X_{u}(\theta)+\Delta^{2} X_{v}(\theta)\right]
$$

Consequently, if one or two of the $u, v$ modes are squeezed, the value $\mathcal{I}_{a^{*}, b^{*}}$ corresponding to maximal correlations is equal to the sum of their squeezing. Experimentally, one has to look for the $u, v$-type modes, a signature of which being that $\mathcal{I}_{u, v}(\theta)$ does not depend on $\theta$ [see (2)], and measure their squeezing (if any). The

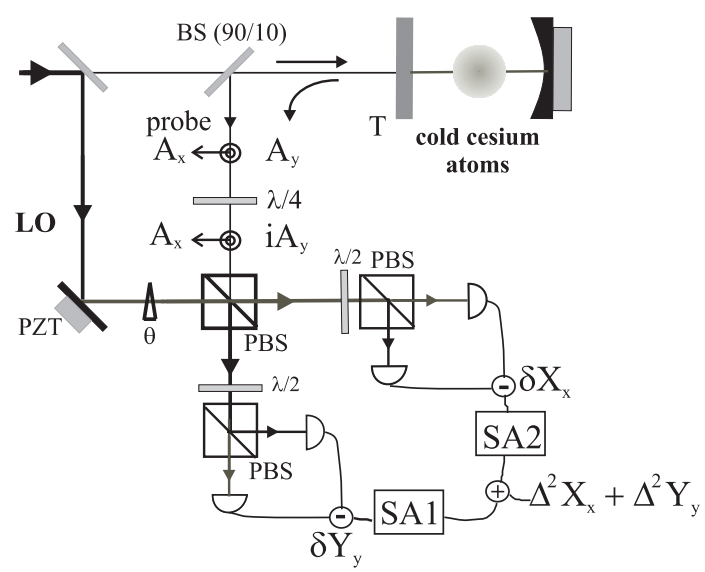

FIG. 1: Experimental set-up: PBS: polarizing beam splitter; $\lambda / 2$ : half-wave plate; PZT: piezo-electric ceramic; SA: spectrum analyzer. $T=0.1$ is the transmission of the cavity coupling mirror. maximally correlated modes are then given by (3) and the amount of their EPR-type correlations by (4).

Let us note that modes $u, v$ are not stricto sensu uncorrelated, since $\left\langle\delta A_{u} \delta A_{v}^{\dagger}\right\rangle$ can be non zero. However, one can think of the correlation properties of modes $a^{*}, b^{*}$ as being created by the mixing of the $u$ and $v$ modes, as it is usually produced by mixing two independent squeezed beams [2, 4, [6]. Let us stress that this analysis provides a general framework for finding out the maximal correlations produced in a two-mode system exhibiting quantum properties. This method is of interest for a class of systems such as the optical parametric oscillator in which the correlations are not produced by mixing independent beams [14].

Coming back to our system, which is symmetrical with respect to the circularly polarized modes $A_{ \pm}$, it is easy to see that $\left\langle\delta A_{x} \delta A_{y}\right\rangle=0$ because $A_{x}$ and $A_{y}$ are combinations with equal weights of $A_{ \pm}$. Since they are squeezed for orthogonal quadratures, one can set $A_{u}=A_{x}$ and $A_{v}=i A_{y}$, which are now squeezed for the same quadrature. Then, using (3), the maximally entangled modes are the $\pm 45^{\circ}$ modes to the $x, y$ basis. This gives us the relevant quantity, $\mathcal{I}_{+45,-45}(\theta)$, which is to be measured. Using $A_{ \pm 45}=\left(A_{x} \pm A_{y}\right) / \sqrt{2}$, the inseparability criterion for the $\pm 45^{\circ}$ modes can be expressed directly in terms of the $x, y$ modes variances with $X_{u}(\theta)=X_{x}(\theta)$ and $X_{v}(\theta)=Y_{y}(\theta)$

$$
\mathcal{I}_{+45,-45}(\theta)=\Delta^{2} X_{x}(\theta)+\Delta^{2} Y_{y}(\theta)<2
$$

When $\theta$ corresponds to the angle $\theta_{s q}$ of the squeezed quadrature of $A_{x}$, both variances are below unity, and $\mathcal{I}_{+45,-45}\left(\theta_{s q}\right)<2$.

In order to experimentally check the inseparability criterion (5), we need to simultaneously measure the fluctuations of $A_{x}$ and $i A_{y}$. After the output of the cavity, we insert a quarter-wave plate that rotates the noise ellipsoid of vacuum mode $A_{y}$ by $\pi / 2$, the beam is mixed on a beamsplitter with a local oscillator (LO), and the two resulting beams are sent into two balanced homodyne detections [Fig. 1]. Thus, we simultaneously measure the quadrature noise of each beam for the same quadrature. The sum of these two signals directly gives the sought quantity $\mathcal{I}_{+45,-45}(\theta)$. In Fig. 2(b), we plot a typical measurement of $\mathcal{I}_{+45,-45}$ as a function of $\theta$, for an analysis frequency of $5 \mathrm{MHz}$. Its minimal value is about 1.9 [1.86 corrected from losses] and corresponds to a case for which $A_{x}$ and $i A_{y}$ are both squeezed by about $5 \%$ [Fig. 2[a)]. Quadrature entanglement is thus achieved in a frequency range given by the cavity bandwidth (3 to $12 \mathrm{MHz}$ ).

Consistently with the general method described above, we also checked that modes $A_{x}$ and $i A_{y}$ correspond indeed to $u, v$-type modes. We therefore measured the quantity $\mathcal{I}_{x, y}$ in a similar manner as $\mathcal{I}_{+45,-45}$, and verified that it is independent of $\theta$ [Fig. 2[(c)], thus proving 

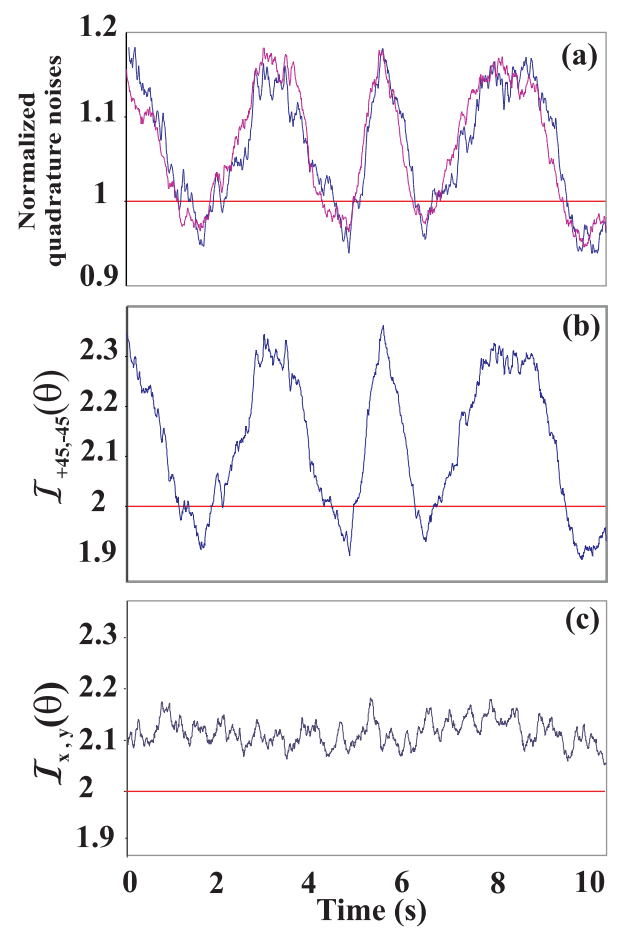

FIG. 2: (a) Quadrature noise spectra of $A_{x}$ and $i A_{y}$, at a frequency of $5 \mathrm{MHz}$, when the relative phase $\theta$ between the LO and the mean field mode is varied in time. (b) Direct measurement of $\mathcal{I}_{+45,-45}(\theta)$. (c) Corresponding measurement of $\mathcal{I}_{x, y}(\theta)$.

that modes $A_{+45}$ and $A_{-45}$ exhibit maximal EPR-type correlations.

Moreover, we note that our measurement not only demonstrates entanglement, but also quantifies it via the entanglement of formation. Following Giedke et al. [10], we introduce the covariance matrix $(\mathrm{CM}) \gamma$ for the $\pm 45^{\circ}$ polarized modes:

$$
\gamma_{i, j}=\left\langle\delta R_{i} \delta R_{j}+\delta R_{i} \delta R_{j}\right\rangle / 2
$$

where $\left\{R_{i}, i=1, \ldots, 4\right\}=\left\{X_{+45}, Y_{+45}, X_{-45}, Y_{-45}\right\}$. Using the fact that $A_{x}$ and $i A_{y}$ are uncorrelated and symmetrical [see Fig. 2(b) and 2(c)], it is straightforward to show that the $\pm 45^{\circ}$ modes have isotropic fluctuations. Choosing $\theta=\theta_{s q}$, the covariance matrix can be expressed in the standard form given in Ref. [10]

$$
\gamma=\left(\begin{array}{cccc}
n & 0 & k & 0 \\
0 & n & 0 & -k \\
k & 0 & n & 0 \\
0 & -k & 0 & n
\end{array}\right)
$$

with $n=\Delta^{2} X_{+45}=\Delta^{2} Y_{ \pm 45}$ and $k=\left\langle\delta X_{+45} \delta Y_{-45}\right\rangle=$ $\left\langle\delta X_{-45} \delta Y_{+45}\right\rangle$ [15]. As calculated by Giedke et al. the EOF $\mathcal{E}$, representing the amount of pure state entanglement needed to prepare our entangled state [16], is then directly related to the inseparability criterion value by
10

$$
\mathcal{E}=f(n-k)=f\left[\mathcal{I}_{+45,-45}\left(\theta_{s q}\right) / 2\right]
$$

with $f(x)=c_{+}(x) \log _{2}\left[c_{+}(x)\right]-c_{-}(x) \log _{2}\left[c_{-}(x)\right]$ and $c_{ \pm}(x)=\left(x^{-1 / 2} \pm x^{1 / 2}\right)^{2} / 4$. For $\mathcal{I}_{+45,-45}=1.86 \pm 0.02$, the EOF is $\mathcal{E}=0.014 \pm 0.003$.

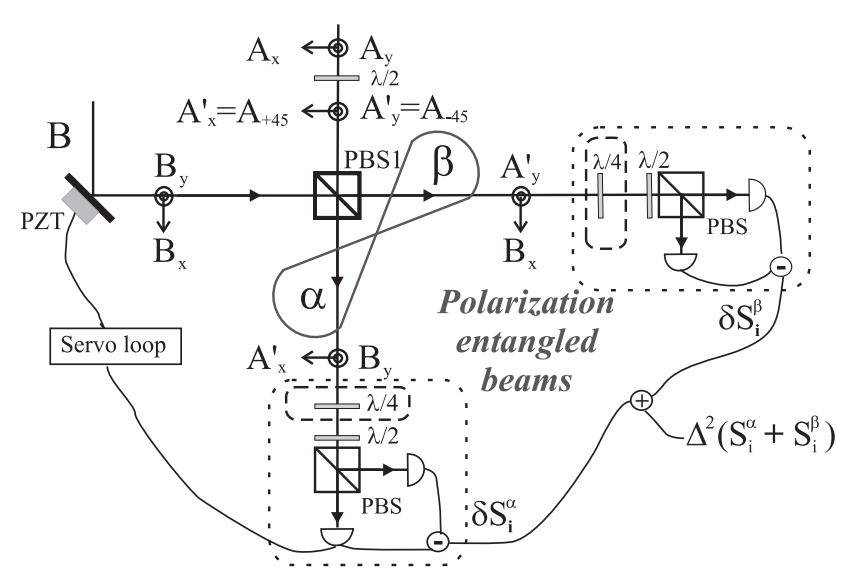

FIG. 3: Set-up for non separable beams generation. Inserting the quarter-wave plates (or not) allows for measuring the fluctuations of $S_{3}^{\alpha}+S_{3}^{\beta}$ (or $S_{2}^{\alpha}+S_{2}^{\beta}$ ). The servo loop is used to lock the $\mathrm{B}$ field phase to the squeezed quadrature angle.

Last, we show that this quadrature entangled beam allows to generate polarization entanglement. Polarization entanglement for two beams $\alpha$ and $\beta$ [5] is achieved when

$\mathcal{I}_{\alpha, \beta}^{S}=\frac{1}{2}\left[\Delta^{2}\left(S_{2}^{\alpha}+S_{2}^{\beta}\right)+\Delta^{2}\left(S_{3}^{\alpha}+S_{3}^{\beta}\right)\right]<\left|\left\langle S_{1}^{\alpha}\right\rangle\right|+\left|\left\langle S_{1}^{\beta}\right\rangle\right|$

where the $S_{i}^{\alpha, \beta}$ are the standard quantum Stokes operators. For this, we produce new modes by mixing the $A_{ \pm 45}$ modes studied previously with additional strong fields. The $A_{ \pm 45}$ modes are obtained from the $x, y$ modes by passing the beam through a half-wave plate with axes at $22,5^{\circ}$. The fields along the $x$ and $y$ directions are now the $A_{+45}$ and $A_{-45}$ fields, that we will denote by $A_{x}^{\prime}$ and $A_{y}^{\prime}$ [see Fig. 3]. The $A_{x}^{\prime}$ and $A_{y}^{\prime}$ are then spatially separated with a polarizing beamsplitter. In the other input of the beamsplitter, we send a strong field $B$ with a polarization at $45^{\circ}$ from the beamsplitter axes, yielding the output fields $B_{y}$ and $B_{x}$. The strong field $B$ is similar to the local oscillator in the previous experiment, except that its phase $\theta_{B}$ is locked to that of one of the $A$ fields by a servo-loop, as shown in Fig. 3] At the two outputs of the beamsplitter, we have two beams $\alpha, \beta$ which are the superposition of, respectively, $A_{x}^{\prime}$ and $B_{y}$, and $A_{y}^{\prime}$ and $B_{x}$. The Stokes operators $S_{i}^{\alpha}$ for one of the outputs are then

$$
\begin{array}{ll}
S_{0}^{\alpha}=A_{x}^{\prime \dagger} A_{x}^{\prime}+B_{y}^{\dagger} B_{y} & S_{1}^{\alpha}=A_{x}^{\prime \dagger} A_{x}^{\prime}-B_{y}^{\dagger} B_{y} \\
S_{2}^{\alpha}=A_{x}^{\prime \dagger} B_{y}+B_{y}^{\dagger} A_{x}^{\prime} & S_{3}^{\alpha}=i\left(B_{y^{\prime}}^{\dagger} A_{x}^{\prime}-A_{x}^{\prime \dagger} B_{y}\right)
\end{array}
$$



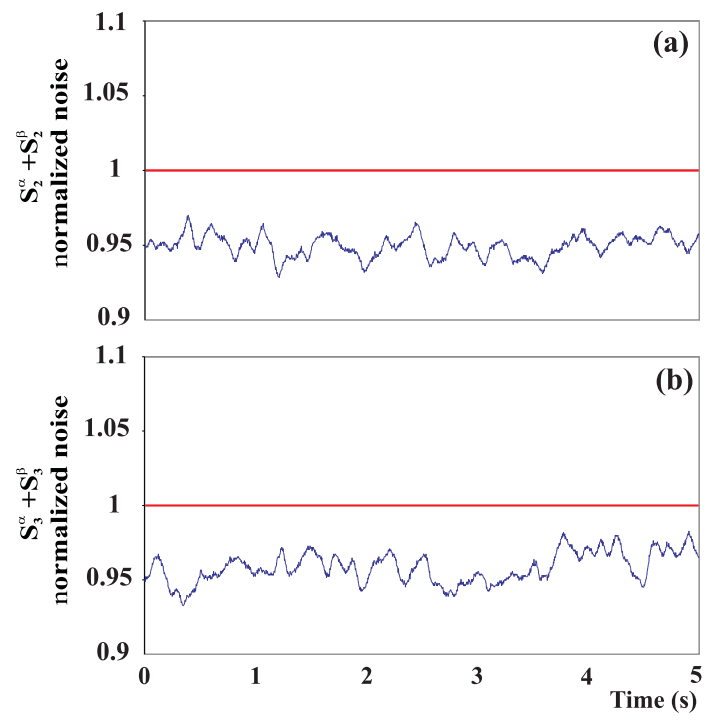

FIG. 4: Normalized noises at $5 \mathrm{MHz}$ of $S_{2}^{\alpha}+S_{2}^{\beta}$ (a) and $S_{3}^{\alpha}+S_{3}^{\beta}$ (b), the phase $\theta_{B}$ being locked with the value of the squeezed quadrature angle $\theta_{s q}$.

The Stokes operators $S_{i}^{\beta}$ for the other output are obtained by exchanging $\mathrm{A}^{\prime}$ and $\mathrm{B}$ in the previous expression. Since the $B$ field is much stronger than the $A$ field, one has $\left|\alpha_{B}\right| \gg\left|\alpha_{A^{\prime}}\right|$, with $\alpha_{B}$ the amplitude of $B_{x}$ and $B_{y}$ and $\alpha_{A^{\prime}}$ the amplitude of $A_{x}^{\prime}$ and $A_{y}^{\prime}$. Then $\left\langle S_{1}^{\alpha}\right\rangle=-\left\langle S_{1}^{\beta}\right\rangle \simeq-\left|\alpha_{B}\right|^{2}$ and the inseparability condition reads

$$
\mathcal{I}_{\alpha, \beta}^{S}<2\left|\alpha_{B}\right|^{2}
$$

In this case the Stokes parameters fluctuations are related to those of the initial $\pm 45^{\circ}$ modes (now denoted $A_{x}^{\prime}, A_{y}^{\prime}$ )

$$
\begin{aligned}
& \delta S_{2}^{\alpha}=\alpha_{B} \delta X_{x}^{\prime}\left(\theta_{B}\right), \quad \delta S_{2}^{\beta}=\alpha_{B} \delta X_{y}^{\prime}\left(\theta_{B}\right) \\
& \delta S_{3}^{\alpha}=-\alpha_{B} \delta Y_{x}^{\prime}\left(\theta_{B}\right), \quad \delta S_{3}^{\beta}=\alpha_{B} \delta Y_{y}^{\prime}\left(\theta_{B}\right)
\end{aligned}
$$

which straightforwardly lead to

$$
\mathcal{I}_{\alpha, \beta}^{S}=\left|\alpha_{B}\right|^{2} \mathcal{I}_{A_{x}^{\prime}, A_{y}^{\prime}}\left(\theta_{B}\right) \equiv\left|\alpha_{B}\right|^{2} \mathcal{I}_{+45,-45}\left(\theta_{B}\right)
$$

The polarization entanglement condition (7) is thus equivalent to the inseparability criterion (15) for the $\pm 45^{\circ}$ modes when $\theta_{B}=\theta_{s q}$. Therefore, quadrature entanglement can be mapped into a polarization basis and lead to polarization entanglement [6]. Experimentally, we use the set-up shown in Fig. 3 and lock the phase of the B field with the squeezed quadrature angle. We then successively measure the $S_{2}$ and $S_{3}$ Stokes operator noises using the appropriate combination [5] of plates and PBS. In Fig. . we present the normalized quadrature noises of $S_{2}^{\alpha}+S_{2}^{\beta}$ and $S_{3}^{\alpha}+S_{3}^{\beta}$ for an analysis frequency of $5 \mathrm{MHz}$. This entanglement between the beams corresponds to a reduction by approximately $5 \%$ in the noise of each variable: $\mathcal{I}_{\alpha, \beta}^{S} /\left|\alpha_{B}\right|^{2}=1.9$, consistently with the quadrature entanglement measurement. From (89), it is also clear that the CM has the same form as ([6).

To conclude, we have reported the generation of continuous variable entanglement via the interaction with cold atoms in cavity. First, we have developed a method to directly measure the inseparability criterion [8] and demonstrated quadrature entanglement between two orthogonally polarized modes. The entanglement was quantified using the entanglement of formation calculated in Ref. 10]. Secondly, we achieve polarization entanglement after mapping the quadrature entanglement onto two spatially separated beams.

This work was supported by the QIPC European Project No. IST-1999-13071 (QUICOV).

[1] C.H. Bennett et al., Phys. Rev. Lett. 70, 1895 (1993); S.L. Braunstein and H.J. Kimble, Phys. Rev. A 61, 042321 (2000); D.P. DiVincenzo, Science 270, 255 (1995), B. Julsgaard et al., Nature 413, 400 (2001).

[2] Z.Y. Ou et al., Phys. Rev. Lett. 68, 3663 (1992).

[3] Y. Zhang et al., Phys. Rev. A 62, 023813 (2000).

[4] C. Silberhorn et al., Phys. Rev. Lett. 86, 4267 (2001).

[5] N. Korolkova et al., Phys. Rev. A, 65, 052306 (2002); N. Korolkova et al., Eur. Phys. J. D 18, 229 (2002).

[6] W.P. Bowen et al., Phys. Rev. Lett. 89, 253601 (2002); W.P. Bowen et al., Phys. Rev. Lett. 90, 043601 (2003).

[7] O. Glöckl et al., J. Opt. B 5, S492 (2003); O. Glöckl et al., Phys. Rev. A 68, 012319 (2003).

[8] L.M. Duan et al., Phys. Rev. Lett. 84, 2722 (2000); R. Simon, Phys. Rev. Lett. 84, 2726 (2000).

[9] V. Josse et al., Phys. Rev. Lett. 91, 103601 (2003).

[10] G. Giedke et al., Phys. Rev. Lett. 91, 107901 (2003).

[11] A. Einstein et al., Phys. Rev. 47, 777 (1935).

[12] V. Josse et al., J. Opt. B 5, S513 (2003).

[13] V. Josse et al., to be published.

[14] L. Longchambon et al., quant-ph/0311123 H. Adamyan and G. Kryuchkyan, quant-ph/0309203 S. Feng and O. Pfister, quant-ph/0310002

[15] The factor $1 / 2$ in our definition for the CM comes from our definition of the quadrature operators.

[16] C.H. Bennett et al., Phys. Rev. A 54, 3824 (1996). 